\title{
Contribution du groupe C.E.A. à la normalisation des matériels pour les installations nucléaires
}

\author{
P. DUMAX et R. SERAN (*)
}

(Manuscrit reçu le 18 janvier 1980)

\begin{abstract}
RÉSUMÉ
Dans les laboratoires de recherche et de production à vocation nucléaire, la normalisation des matériels contribue efficacement à la rentabilité des installations et à la protection des individus et des biens. L'action de normalisation des moyens techniques de Protection, de Manipulation, de Détection et de Sécurité (dite P.M.D.S.), engagée par le C.E.A. en 1967, est actuellement poursuivie au sein de l'Institut de Protection et de Sûreté Nucléaire par le Service Technique d'Equipements de Protection et de Dosimétrie implanté au C.E.N./Saclay. Celui-ci s'applique d'une part, à établir des documents de normalisation intérieurs à l'entreprise et d'autre part, à collaborer aux travaux des organismes officiels normalisateurs. Après un rappel des avantages bien connus qu'apporte la normalisation, on fait le point des travaux réalisés par les groupes de travail intercentres du C.E.A. et on cite les publications diffusées et à venir.
\end{abstract}

\begin{abstract}
In research and production laboratories working in the nuclear field standardization of equipments greatly improves the profits of the installation and the protection of individuals and goods. The standardization effort on technical equipments of shielding, handling, detection and safety (so called P.M.D.S.) initiated by the C.E.A. in 1967 is being carried out now within the Institute of protection and nuclear safety (I.P.S.N.) by a technical service specialized in protection and dosimetry (S.T.E.P.D.). Its purpose is to establish standardization documents for internal use and to take part in the work of official standardization organizations. The benefits of standardization are reviewed. The achievements of the various working groups common to C.E.A. centers are stated and the documents published or to be published are listed.
\end{abstract}

(*) Commissariat à l'énergie atomique, I.P.S.N., Département de protection, Services techniques de protection, Service technique d'équipements de protection et de dosimétrie, B.P. $\mathrm{n}^{\circ} 2,91190$ Gif-sur-Yvette. 
Du fait du développement des recherches sur l'énergie atomique et des applications relatives à cette forme d'énergie, des laboratoires de haute activité ont été construits dans de nombreux pays. On a cependant constaté que pour faire face aux problèmes concernant les principes, les méthodes et les procédés intervenant dans ces installations, concepteurs et exploitants ont, souvent, arbitrairement implanté un grand nombre d'équipements spécifiques. Dans bien des cas, cet aménagement anarchique d'enceintes blindées, d'enceintes de confinement et de leurs moyens de manipulation, de vision, de transfert, etc. a grevé lourdement le budget d'exploitation de ces installations et a parfois nui à la sécurité des travailleurs.

Sur les plans technique et financier, l'expérience a montré que la rentabilité et la sûreté d'une installation, nucléaire ou non, passe par l'unification des matériels et des composants, par l'exploitation cohérente de ces matériels unifiés, c'est-à-dire par un effort concerté de réflexion entre les concepteurs, les industriels fabricants et les exploitants, ce qui nous conduit à la notion de normalisation.

Par définition, normaliser c'est soumettre une production à un ensemble de règles techniques, résultant de l'accord entre producteurs et utilisateurs, pour réduire le nombre de types d'un même article afin d'abaisser leur prix de revient et de rendre les produits uniformes et leurs composants interchangeables. Mais une normalisation efficace se doit d'aller plus loin : elle permettra non seulement d'organiser rationnellement les fabrications de la matière première au produit fini, mais aussi de fixer les règles de construction, de contrôle de qualité, d'exploitation et d'entretien des appareillages. Néanmoins, ces spécifications centralisées au niveau national ou international ne devront pas conduire à des normes invariables, mais plutôt définir les buts à atteindre et respecter la liberté d'invention, voire susciter les désirs de réalisation des producteurs.

D'une façon générale, la normalisation des matériels intéresse le concepteur, l'industriel fabricant et l'exploitant :

Le concepteur voit son travail grandement simplifié par l'existence de documents techniques et de normes correspondantes. L'utilisation de normes de forme, de calcul, de contrôle de qualité, de classification de caractéristiques lui permet de prendre en compte un grand nombre de facteurs opératoires et ainsi d'améliorer la conception d'installations industrielles. Il est également important de noter que le choix d'appareillages normalisés facilite l'élaboration des dossiers de sûreté et simplifie l'obtention des autorisations réglementaires par les Services administratifs compétents.

L'industriel fabricant est également intéressé par la normalisation. Une fabrication bien pensée, bien préparée, lui permettra de produire des matériels en série et, ainsi, d'amortir les dépenses totales engagées et d'atteindre des prix plus intéressants. Ces séries de matériels normalisés pourront plus facilement subir les contrôles aux différents stades de leur fabrication. Les améliorations technologiques éventuelles seront plus aisées. 
Pour l'exploitant de laboratoire, le résultat de cet effort de normalisation se traduit par un meilleur rendement de l'exploitation et une sécurité accrue. L'unification des équipements apporte des possibilités d'entretien élargies, une formation du personnel commune et des possibilités d'interchangeabilité des équipements et de modification des installations.

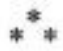

L'action de normalisation, entreprise au C.E.A. en 1967 pour définir les moyens techniques à utiliser dans les installations nucléaires pour assurer la protection individuelle et collective du personnel, est actuellement poursuivie au sein de l'Institut de Protection et de Sûreté Nucléaire (I.P.S.N.) par le Service technique d'équipements de Protection et de Dosimétrie (S.T.E.P.D.) implanté au C.E.N./Saclay. Cette action dite de Normalisation des Matériels et Équipements de Protection, de Manipulation, de Détection et de Sécurité (Normalisation P.M.D.S.) est exercée à l'usage des personnes qui envisagent d'étudier, de construire ou de modifier des laboratoires de recherche ou de production à vocation nucléaire. Elle couvre notamment les domaines suivants :

- constitution d'enceintes blindées ou de confinement, de boîtes à gants et de hottes ventilées...;

- aménagement de ces enceintes (fig. 1) (systèmes de vision, de manipulation, de transfert des produits; ventilation, filtration; circuits électriques et de fluides...);

- contrôles de qualité, homogénéité des blindages...;

- aménagement des zones de travail (peintures et revêtements; détection des atmosphères dangereuses, des inondations; détection, extinction incendie...);

- protection du personnel (vêtements de protection et d'intervention, détection et mesure des rayonnements...);

- décontamination du matériel...

Pour ce faire, le S.T.E.P.D. encourage et développe la formation de groupes de travail pour promouvoir la coopération technique entre les agents du Groupe C.E.A. par des échanges d'informations ainsi que par la collection et la diffusion de données concernant le travail en milieu hostile. Au niveau intercentres, l'organe directeur du programme P.M.D.S. est la « Commission des Présidents P.M.D.S. " dont les membres sont désignés par les Directeurs des Centres et Êtablissements du Groupe C.E.A. Cette commission a notamment pour rôle de coordonner les activités des groupes de travail internes à l'entreprise et de déterminer la priorité des sujets à traiter.

Les groupes de travail sont principalement formés d'agents qui appartiennent aux unités scientifiques et de support technique dont les activités sont centrées sur les problèmes inhérents aux installations nucléaires : construction, exploitation, protection, entretien, décontamination... Pour accroître le champ de ces échanges d'expérience, certains collaborateurs extérieurs acceptent de participer à ces travaux.

vol. $15-\mathrm{N}^{0} 2$ 


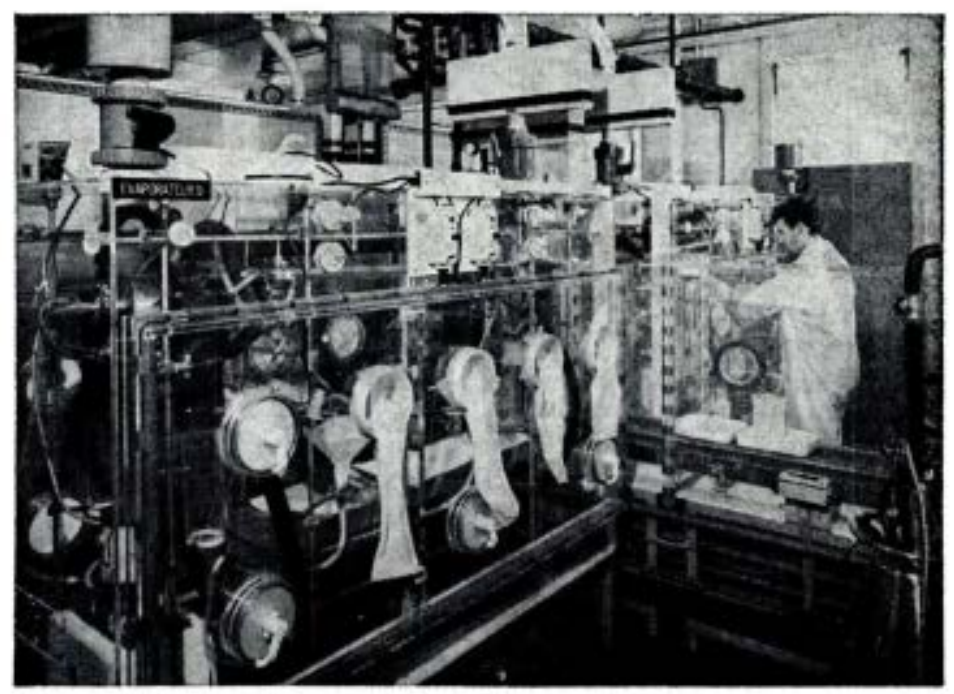

Fig. 1. - Chaine de boites à gants (Laboratoire de haute activité, C.E.N./Saclay). Ronds de gants, obturateurs, filtres..., sont normalisés, de même que les circuits de fluides et les circuits électriques.

Ces spécialistes visent évidemment à obtenir tous les avantages économiques et techniques de la normalisation de l'industrie classique, mais, de surcroît, après enquête éventuelle sur le comportement en service des matériels, recommandent les meilleurs méthodes, procédés et équipements qui répondent aux impératifs des travaux en ambiance nucléaire, sous les trois aspects de la rentabilité, de la fiabilité et de la sécurité.

Depuis 1967, leurs travaux ont donné lieu à une publication de documents techniques tout particulièrement destinés aux secteurs professionnels suivants :

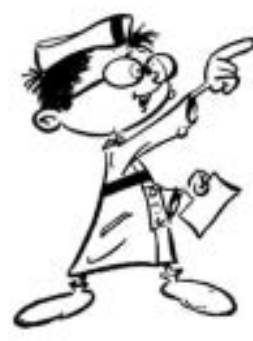

Dans le Groupe C.E.A. :

- concepteurs, constructeurs et utilisateurs d'installations nucléaires:

- services ou commissions concernés par la Sécurité en ambiance nucléaire (Services de protection contre les radiations, Formations locales de sûreté, ingénieurs de sécurité, Médecine du travail, Commissions d'hygiène et de sécurité...);

- filiales du Groupe C.E.A. [COGEMA $\left({ }^{1}\right)$ ECOPOL S.A., S.T.M.I. $\left({ }^{2}\right)$ SOCATRI $\left.\left({ }^{3}\right) \ldots\right]$.

(1) COGEMA, Compagnie Générale des Matières Nucléaires.

(2) S.T.M.I., Société de Travaux en Milieu Ionisant.

(3) SOCATRI, Société Auxiliaire du Tricastin. 


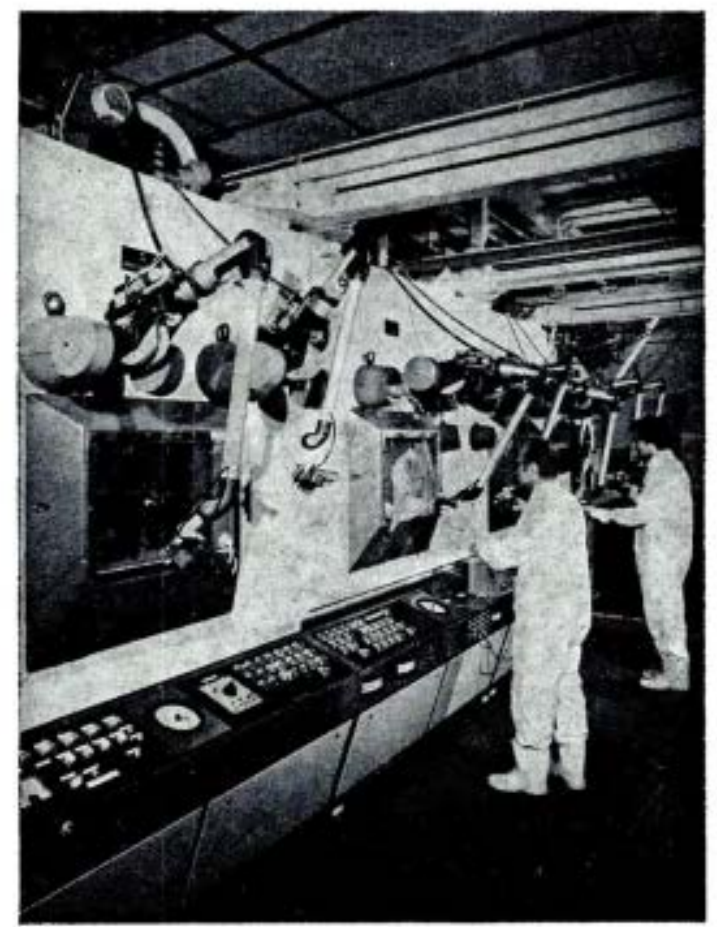

Fig. 2. - Télémanipulation dans une installation de radiochimie par manipulateur maitre-esclave type MA II. (Ce manipulateur normalisé a été vendu sous licence C.E.A. dans 17 pays.)

Hors C.E.A., en France et à l'étranger : hôpitaux...);

- industriels et exploitants de laboratoire (EDF $\left({ }^{1}\right)$, universités,

- sociétés d'ingénierie;

- associations corporatives de l'industrie nucléaire;

- organismes officiels de normalisation;

- journalistes spécialisés...

Dans le cadre de la « Normalisation des Matériels et des Équipements de Protection, de Manipulation, de Détection et de Sécurité n, le S.T.E.P.D. élabore et diffuse, dans la collection P.M.D.S., trois types de documents concernant les moyens techniques à utiliser dans les laboratoires de recherche et de production à vocation nucléaire :

- le catalogue intercentres P.M.D.S. qui précise non seulement les positions adoptées par les «Commissions des Stocks et des Matériels " en

(1) E.D.F., Électricité de France. 


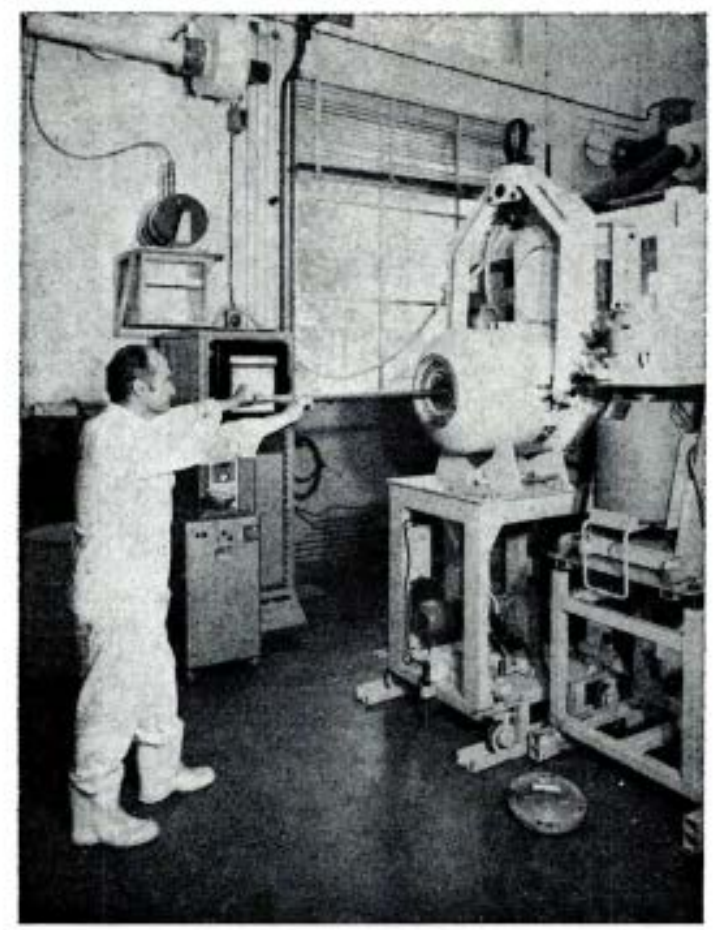

Fig. 3. - Système PADIRAC. Ce conteneur de transport, étanche et blindé, permet l'introduction et l'évacuation de matières radioactives solides.

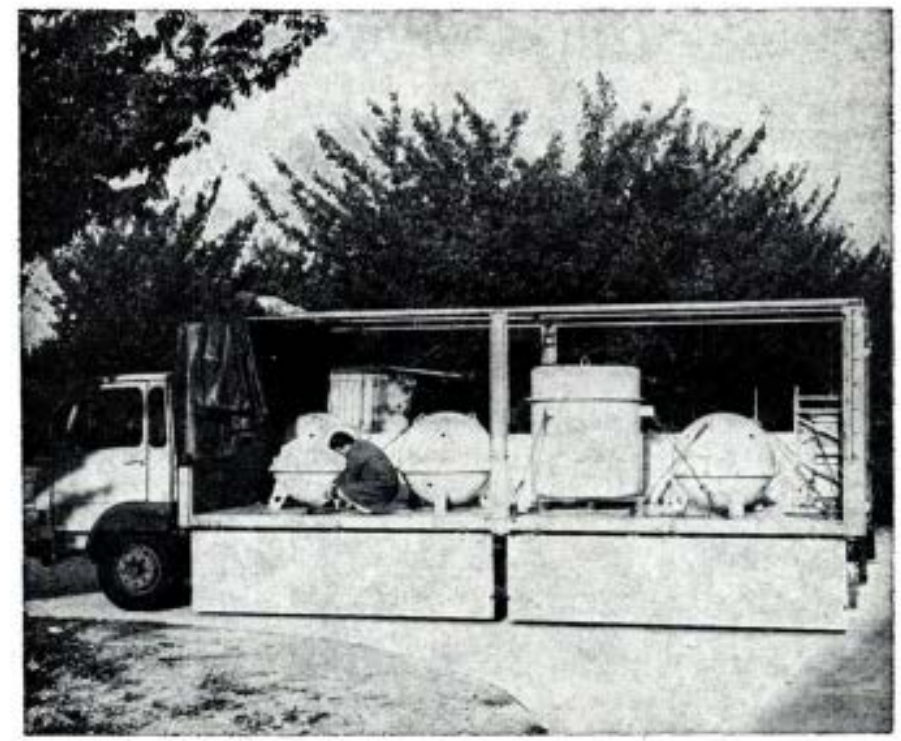

Fig. 4. - Camion aménagé pour le transport

des systèmes CENDRILLON et PADIRAC dans leur coque antichoc et antifeu. 
ce qui concerne les approvisionnements prévus par le Groupe C.E.A. mais présente surtout de nombreuses informations sur le "savoir faire " et les équipements sélectionnés;

- des notes techniques spécifiques qui recommandent avec précision l'utilisation de certains procédés et matériels;

- des fascicules d'information P.M.D.S. destinés à faire rapidement connaître aux responsables d'installations les nouveautés techniques proposées par les constructeurs et les exploitants.

La forme et la teneur de cette documentation, destinée aux divers services du Groupe C.E.A. comme aux organismes extérieurs, sont développées ci-après :

$1^{\circ}$ Catalogue intercentres de Protection-Manipulation-Détection-Sécurité (P.M.D.S.).

Ce document présente une sélection du matériel normalisé employé dans le Groupe C.E.A. pour :

- la construction d'enceintes blindées et/ou étanches;

- la manipulation dans ces enceintes (fig. 2);

- les transferts et les tranports ( fig. 3 et 4 );

- la protection et le contrôle individuel et collectif.

Pour chaque famille d'équipements, ce document présente un synoptique d'ensemble des matériels et des tableaux de nomenclature, ce qui permet de faciliter la gestion de ces matériels à l'intérieur des centres et éventuellement leur transfert d'un centre à l'autre. Des pages de renseignements techniques donnent des conseils sur le choix, la classification ou l'utilisation des différents matériels, ainsi que les caractéristiques, le mode d'emploi et le montage de ces équipements. Des pages de détails illustrent chacun des matériels et donnent des renseignements particuliers d'utilisation. Enfin, quand c'est nécessaire, dans des pages de remarques importantes, on peut trouver, à titre d'information, des matériels sélectionnés mais non détaillés dans le document ou des annonces de matériels nouveaux en cours d'industrialisation. Une table des matières et une liste de fournisseurs complètent chaque fascicule.

Les chapitres déjà parus sont les suivants (*):

Chapitre I: Éléments constitutifs d'enceintes blindées (paru en 1968).

Chapitre $I I$ : Éléments constitutifs d'enceintes de confinement. $1^{\text {re }}$ partie : ronds de gants, bagues d'enceintes, canons d'éjection, accessoires divers pour boites à gants (1969. rédédition prévue en 1982; remise à jour en cours).

(†) Comment se procurer le catalogue P.M.D.S. :

- pour les agents C.E.A. : aux Magasins des centres ou auprès des correspondants P.M.D.S.

- pour les organismes extérieurs : à la Société ETAI, 20-22, rue de la Saussière, 92100 Boulogne-Billancourt. Téléphone : 604.81.13.

voL. $15-\mathrm{N}^{\circ} 2$ 

(1969).

Chapitre III : Manipulation. $1^{\text {re }}$ partie : gants, pinces à distance et soufflets d'étanchéité

Chapitre IV : Enceintes de confinement et éléments de transfert. $1^{\text {re }}$ partie : hottes, boites à gants, ventilation, filtration (1974).

Chapitre VII : Vêtements de protection et équipements individuels d'intervention en milieu hostile. $1^{\text {re }}$ partie : vêtements ordinaires de travail, vêtements et équipements de sécurité, tenues spéciales et d'intervention (1976). $2^{2}$ partie : vêtements ventilés, accessoires de vêtements ventilés, appareils de protection des voies respiratoires et accessoires (1978).

Sont en préparation :

Chapitre II : Éléments constitutifs d'enceintes de confinement. $2^{\circ}$ partie : équipements pour circuits de fluides, équipements pour circuits électriques, équipements de sécurité (1982).

Chapitre III : Manipulation. $2^{*}$ partic : manipulateurs maitre-esclave, accessoires pour manipulateurs (1982).

Chapitre IV : Enceintes de confinement et éléments de transfert. $2^{\circ}$ partie : éléments de transfert, portes-sas, tunnels de liaison, transferts de solides et de liquides (1982).

Chapitre VIII : Protection contre l'incendie, protections diverses. $1^{\text {re }}$ partic : détection incendie, extinction incendie (1980). $2^{e}$ partie : détection des atmosphères dangereuses, peintures et revêtements, équipements structurels contre l'incendie (1981).

\section{$2^{\circ}$ Notes techniques P.M.D.S.}

Ces notes techniques ont essentiellement pour objet de donner des conseils aux responsables des laboratoires à vocation nucléaire qui utilisent des procédés ou des matériels normalisés. Elles complètent les caractéristiques et le " savoir faire " décrits dans les pages de renseignemnts techniques du catalogue P.M.D.S. Ces notes sont rédigées par des groupes de travail formés de spécialistes des différents établissements du groupe C.E.A. qui, avec l'accord de leur hiérarchie, se sont portés volontaires pour coopérer aux travaux de normalisation P.M.D.S.

\section{Sont parues :}

(1) Manipulateurs maitre-esclave : par la Section Équipement pour Milieu Hostile (DPr/STEP/EMH), novembre 1972, 24 pages (document épuisé, remise à jour en cours).

(2) Caractéristiques générales des revétements par peintures recommandés dans les laboratoires de haute activité : par le Groupe de travail intercentres $\alpha$ Peintures et revêtements $x$ Note technique $\mathrm{n}^{\circ} 1$, avril 1973, 96 pages (usage interne au C.E.A., document épuisé, remise à jour en cours).

(3) Transfert, stockage et transport de liquides radioactifs par récipients de type "Cendrillon " : par la Section Équipement pour Milieu Hostile (DPr/STEP/EMH), janvier 1975, 90 pages (en réimpression).

( 3 bis) Version anglaise (février 1976, 93 pages).

(4) Conseils pour la mise en auvre des peintures dans les installations nucléaires : par le Groupe de travail intercentres « Peintures et revêtements $n$ Note technique $n^{*} 5$, octobre 1975, 93 pages (document épuisé, remise à jour en cours). 
(5) Généralités concernant les atmosphères dangereuses, toxiques et explosives : par le Groupe de travail intercentres « Détection des Atmosphères Dangereuses $»$ Note technique $n^{\circ} 1$, décembre 1977, 35 pages.

A paraitre :

Décontamination radioactive du matériel.

Manipulateurs maitre-esclave ( ${ }^{\circ}$ édition, mise à jour et compléments).

Revêtements par peinture dans les installations nucléaires ( $2^{e}$ édition, refonte et mise à jour des notes techniques).

\section{$3^{\circ}$ Fascicules d'information P.M.D.S.}

Ces fascicules semestriels, rédigés par le S.T.E.P.D. à partir des informations qui lui ont été communiquées par les techniciens du Groupe C.E.A., sont destinés à faire connaitre rapidement aux utilisateurs des laboratoires de haute activité comme aux commissions P.M.D.S., les nouveaux équipements ou procédés qui peuvent être mis en cuvre dans les installations nucléaires. Le dernier numéro (5 décembre 1979) a été adressé à 350 personnes. Ce document est diffusé sur demande.

Bien entendu, le S.T.E.P.D. souhaite recevoir de nombreux articles pour ses prochaines publications. Toutes observations et suggestions qui pourraient améliorer la forme et la teneur de ce document seront les bienvenues.

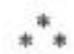

Outre cette action de normalisation P.M.D.S. interne au groupe C.E.A., le S.T.E.P.D. représente les intérêts du Commissariat auprès des organismes professionnels et des associations de normalisation U.N.M. $\left({ }^{1}\right)$, AFNOR $\left({ }^{2}\right)$, C.E.N. $\left({ }^{3}\right)$, I.S.O. $\left({ }^{4}\right)$.

Un comité de sélection des matériels d'équipement pour milieux hostiles a été créé en 1972. Il réunit des industriels (Groupement Intersyndical de I'Industrie Nucléaire, G.I.I.N.) ${ }^{5}$ ), des membres de diverses unités opérationnelles du C.E.A., de l'AFNOR et d'Électricité de France.

Les normes, qu'elles soient nationales ou internationales, s'établissent avec une grande prudence. Toutefois, nous noterons avec satisfaction que les documents élaborés au Commissariat à l'Énergie Atomique ont permis l'établissement de normes françaises AFNOR, notamment sur les éléments

(') U.N.M., Union de Normalisation de la Mécanique.

${ }^{2}{ }^{2}$ AFNOR, Association Française de Normalisation.

(3) C.E.N., Comité Européen pour la Coordination de la Normalisation.

$\left({ }^{4}\right)$ I.S.O., International Organization for Standardization.

(5) Les travaux à charge du G.I.L.N. ont été repris fin 1979 par le SYGECAM/G.E.N. SYGECAM/C.E.N., Syndicat Général des constructeurs d'équipements pour la chimie, les matières plastiques et le eaoutchouc, l'alimentation et les industries diverses/Groupe des équipements nucléaires.

VOL. $15 \cdot \mathrm{N}^{0} 2$ 
de blindage; ce sujet fait, actuellement, l'objet de travaux dans le cadre de 1'I.S.O. Des normes françaises sur la ventilation et la filtration, la conception des enceintes étanches, le contrôle et la classification des enceintes de confinement, la classification des laboratoires en zones sont également parues ou en cours d'élaboration. Tout ce travail est le fruit d'une politique de normalisation que le C.E.A. et des industriels poursuivent ensemble, depuis près de 20 années, avec le soutien des principaux utilisateurs.

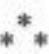

Nous avons vu que, potentiellement, un équipement normalisé apporte avec lui sa sécurité propre et celle d'un emploi connu jusqu'aux consignes précises d'utilisation. Dans l'implantation et le maintien de cette normalisation, de grands efforts doivent porter sur la réalisation et la diffusion de documents techniques, la persuasion des utilisateurs qui adoptent, trop souvent par ignorance, des solutions non normalisées et, surtout, l'évolution permanente des matériels et la formation du personnel à leur utilisation. En agissant de cette manière, nous faisons ce qui est en notre pouvoir pour que notre participation soit utile à tous.

On constate, ainsi, que tant au C.E.A. qu'au niveau de l'industrie, en France comme à l'étranger, on admet qu'une part croissante des efforts doit porter, maintenant, sur ce domaine de la normalisation; ce travail se justifie par une acquisition de connaissances très importante et par une économie potentielle que ces études générales permettent de faire sur des études particulières à venir. Nous trouvons là une chance de profiter en même temps de toute l'expérience d'exploitation actuelle et des meilleures idées des laboratoires ou des industriels constructeurs, le tout modéré par le souci commun de faire l'économie d'une prolifération d'études ou de réalisations inutiles et le besoin d'atteindre une sûreté sans cesse améliorée.

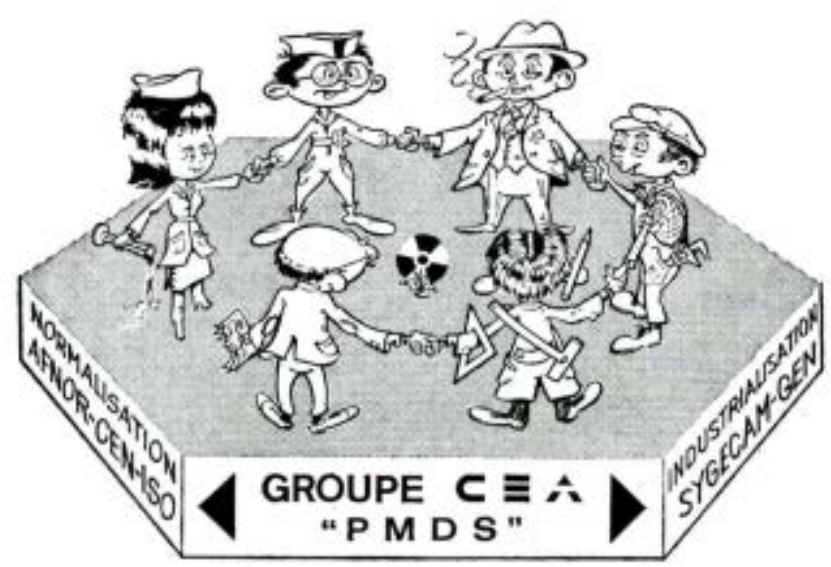

RADIOPROTECTION 